\title{
De l'information à la compréhension : une affaire de spécialistes
}

\author{
From Information to Understanding: A Matter of Specialists
}

\author{
T. Desmettre \\ C SFMU et Lavoisier SAS 2017
}

En France, comme dans d'autres pays occidentaux, le premier reproche adressé aux médecins est résumé dans la formule : « il ne m'a rien dit », ce qui signifie le plus souvent : « il ne m'a pas dit ce que j'attendais de lui » ou « je n'ai pas compris ce qu'il m'a dit ». Ce défaut d'information est la cause la plus fréquente des procédures engagées contre un médecin. Le code de la Santé Publique est très clair sur ce point, il précise que «le médecin doit à la personne qu'il examine, qu'il soigne ou qu'il conseille, une information loyale, claire et appropriée sur son état, les investigations et les soins qu'il lui propose. (...) [I]l tient compte de la personnalité du patient dans ses explications et veille à leur compréhension »[1]. Mais informer n'est pas suffisant : délivrer un message est une chose, se faire comprendre et s'assurer de la bonne compréhension en est une autre ; c'est simplement vérifier que ce que l'on pense avoir bien fait l'est effectivement. Il n'est peut-être pas inutile de rappeler que le terme « docteur » vient du latin docere qui signifie « enseigner, instruire », ce qui implique simplification, répétition, échange. S'il peut être difficile de faire comprendre une situation complexe, il faudra dans ce cas simplifier le discours, s'assurer que le patient saisit ce qu'on lui explique, lui laisser poser des questions complémentaires, en allant même jusqu'à lui demander de reformuler ce qu'il a compris.

Dans ce numéro des Annales Françaises de Médecine d'Urgence, Sanchez et al. [2] présentent les résultats d'une étude observationnelle qui étudie, au cours d'un rappel téléphonique de patients laissés sur place en SMUR, ce que ceux-ci avaient compris des informations communiquées par le médecin [2]. Les champs explorés concernent le diagnostic, les soins et traitements, les éléments de surveillance et de suivi et enfin les conseils en cas de récidive ou d'aggravation. Il en ressort que dans $81 \%$ des cas, au moins un des domaines montre un déficit de compréhension, plus par-

T. Desmettre $(\bowtie)$

Service d'accueil des urgences/SAMU 25, université de Bourgogne Franche Comté, UMR 6249 UFC/CNRS, centre hospitalier universitaire de Besançon, 1 boulevard Fleming, F25030 Besançon cedex, France

e-mail : thibaut@desmettre.org ticulièrement celui concernant le suivi préconisé et les motifs de rappel au centre 15. Les facteurs associés à une moins bonne compréhension sont un âge avancé, le caractère aigu de la pathologie et les modalités de la communication de la part du médecin.

Malgré son faible effectif et son caractère monocentrique, cette étude nous interpelle à plusieurs titres. Tout d'abord, face à l'importance de cette question de la compréhension, il est surprenant que très peu de travaux aient été publiés dans ce domaine pour la médecine d'urgence préhospitalière. Ce manque de travaux est étonnant, d'autant que l'on connaît dans notre discipline l'importance de l'incertitude dans nos prises de décision et l'importance du risque que l'on prend quand les patients sont laissés sur place en SMUR. Ce risque est, de fait, en partie lié à l'absence de temps de mise en observation qui permettrait la confirmation ou l'infirmation du ou des diagnostics évoqués ou réfutés. Dès lors, des garde-fous sont indispensables, il peut s'agir de l'information des proches. Si de nombreux travaux ont été réalisés en réanimation en ce qui concerne l'information et la compréhension des familles, là encore la littérature est pauvre pour la médecine d'urgence préhospitalière. Dans un travail multicentrique publié en 2012, Debaty et al. [3] avaient montré que la moitié des familles de patients pris en charge en SMUR n'avaient pas compris les informations relatives à la gravité de leur parent.

Comment faire mieux ? Face à ces risques, la maîtrise des techniques de communication est essentielle. Malgré les contraintes et compte tenu des enjeux, il faut se donner le temps de l'information du patient, de ses proches, le temps de vérifier la bonne compréhension des informations et des conseils. La reformulation permet de s'en assurer, elle fait partie des recommandations au centre 15, pour les appelants. Elle peut s'appliquer également chaque fois que nécessaire pour des patients du SMUR. Dans leur étude, Sanchez et al. [2] précisent que le centre 15 de Strasbourg a mis en place ce rappel systématique des patients laissés sur place en SMUR, ce qui semble indispensable afin d'assurer un feed-back de ces interventions. On peut d'ailleurs s'interroger sur l'intérêt de cette procédure pour certains patients quittant le service 
des urgences, chez lesquels on estime que le niveau de compréhension et de suivi des conseils est ou sera faible.

L'autre volet concerne bien sûr la formation à la communication et l'utilisation des outils de communication. Cette formation sera intégrée dans la maquette du diplôme d'études spécialisées (DES) de médecine d'urgence, elle pourra faire appel à la simulation, aux jeux de rôle, aux séances d'apprentissage au raisonnement clinique. Cette formation doit être adaptée aux spécificités de notre spécialité autour de la compréhension des patients et/ou de leurs proches dans le contexte singulier de l'urgence préhospitalière mais également intrahospitalière. Compte tenu des enjeux, une attention spécifique doit être apportée à nos futurs jeunes internes sur ce point de leur formation : c'est en effet une affaire de spécialistes, la nôtre. Elle est bien en phase avec la nouvelle toute récente de la création à la rentrée prochaine d'une soussection de médecine d'urgence (48-05) au sein du Conseil National des Universités, dernière étape nécessaire à l'établissement d'une spécialité à part entière [4].

Liens d'intérêts : L'auteur déclare ne pas avoir de lien d'intérêt.

\section{Références}

1. République française (2012) Article R4127-35 modifié par décret n $2012-694$ du 7 mai 2012 - art. 2 portant modification du code de déontologie médicale. https://www.legifrance.gouv.fr/affichCodeArticle.do;jsessionid=EDC72BA3435F456473B1995E233DE6F1.tpdila16v_2?cidTexte=LEGITEXT000006072665\&idArticle $=$ LEGIARTI000025843586\&dateTexte $=20170518 \&$ categorieLien=id\#LEGIARTI000025843586 (dernier accès le 18 mai 2017)

2. Sanchez A, Bejinariu L, Schaeffer M, Pellacia T (2017) Que comprennent les patients laissés sur place par le SMUR quant aux informations communiquées par le médecin ? Ann Fr Med Urg 7:159-65

3. Debaty G, Ageron FX, Minguet L, et al (2015) More than half the families of mobile intensive care unit patients experience inadequate communication with physicians. Intensive Care Med 4:1291-8

4. République Française (2015) Arrêté du 27 avril 2017 modifiant l'arrêté du 29 juin 1992 fixant la liste des sections, des soussections et des options ainsi que le nombre des membres de chaque section et sous-section des groupes du Conseil national des universités pour les disciplines médicales, odontologiques et pharmaceutiques. JORF $\mathrm{n}^{\circ} 0106$ du 5 mai 2017. https://www.legifrance.gouv.fr/eli/arrete/2017/4/27/MENH1712826A/jo/texte (Dernier accès le 18 mai 2017) 